\title{
A INFLUÊNCIA DO EMPENHAMENTO ORGANIZACIONAL E PROFISSIONAL DOS ENFERMEIROS NAS ESTRATÉGIAS DE RESOLUÇÃO DOS CONFLITOS
}

\author{
Paula Pinho* \\ Carlos Albuquerque ${ }^{\dagger}$
}

\begin{abstract}
RESUMO: As mudanças na área da saúde e o conjunto de alterações estruturais na profissão e carreira de enfermagem interferem na dinâmica e estabilidade do futuro dos enfermeiros.

O objetivo da investigação centrou-se no estudo da influência do empenhamento organizacional e profissional dos enfermeiros nas estratégias de resolução de conflitos.

Trata-se duma pesquisa não experimental de natureza quantitativa e transversal, seguindo uma via descritivo-correlacional. Os dados foram obtidos através da aplicação de um questionário a 102 enfermeiros a exercer funções em diferentes tipos de unidades de Cuidados de Saúde Primários, existentes nos Agrupamentos de Centros de Saúde da Região Dão-Lafões, sendo a amostra de conveniência, na maioria do sexo feminino (82,4\%), com idade média de 39,33 anos. Como instrumentos de medida utilizámos três escalas que agrupam um total de nove subescalas que pretendem avaliar: o empenhamento organizacional, através do modelo adaptado de Meyer e Allen, " Organizational Commitment Questionnaire”, o empenhamento profissional, através "Attitudinal Commitment Scale", desenvolvida por Langford e as estratégias de resolução de conflitos através do "Rahim Organizational Conflict Inventory - II", constituído por 28 itens, que pretende medir a forma como os indivíduos lidam com as situações conflituais perante superiores (Forma A), subordinados (Forma B) e colegas (Forma C).

A maioria dos enfermeiros apresenta scores moderados/elevados de empenhamento organizacional e de empenhamento profissional. Confirmam-se as hipóteses de que algumas variáveis sociodemográficas e profissionais influenciam as estratégias de resolução de conflitos adotadas pelos enfermeiros. Também, o empenhamento organizacional e profissional estão positivamente
\end{abstract}

\footnotetext{
* Enfermeira da Unidade de Saúde Familiar Lusitana

${ }^{\dagger}$ Docente do Ensino Superior Politécnico, Escola Superior de Saúde de Viseu
} 
correlacionados entre si e influenciam as estratégias de resolução de conflitos adotadas pelos enfermeiros.

Espera-se que gestores e colaboradores fomentem e sejam preparados para gerir os conflitos de forma adequada, para que estes se tornem um motor de inovação e desenvolvimento dentro das organizações.

Palavras-chave: Empenhamento Organizacional, Empenhamento Profissional, Estratégias de Resolução de Conflitos, Enfermeiros.

\begin{abstract}
The changes in the health area and the set of structural changes in the nursing profession and career interfere in the dynamics and stability of the future of the nurses. The aim is to study the influence of organizational and professional commitment of the nurses in the strategies of conflict resolution.

This is a quantitative, transversal and non-experimental research, following a descriptive-correlational way. Data were obtained by applying a questionnaire to 102 nurses that work on different types of Primary Health Care Units, of the various Health Centres of Lafões Area. The convenience sample is mostly female (82,4\%), with the average age of 39,33 years. As measuring instruments we used three scales that grouped a total of nine subscales used to evaluate: the organizational commitment, through the model adapted from Meyer and Allen (1997), "Organizational Commitment Questionnaire," the professional commitment through "Attitudinal Commitment Scale" developed by Langford (1979) and the strategies of conflict resolution through the - "Rahim Organizational Conflict Inventory - II", formed by 28 items, which aims to measure how individuals deal with conflict situations towards their superiors (Form A), their subordinates (Form B) and their colleagues (Form C).

The majority of nurses present moderate / high scores of organizational commitment and of Professional commitment.

The study confirmed the hypothesis that certain sociodemographic and professional variables influence the strategies of conflicts adopted by nurses. Also, the organizational and professional commitments are positively correlated with each other and they influence the strategies of conflicts adopted by nurses.

It is expected that managers and collaborators are prepared to promote and manage conflicts appropriately, so that they can become a motive of innovation and development within organizations.
\end{abstract}

Keywords: Organizational Commitment, Professional Commitment, conflict resolution strategies, Nursing. 


\section{INTRODUÇÃO}

As políticas de ajuste estrutural que se aplicam fazem com que a reforma do setor da saúde seja vista como uma prioridade devido aos imperativos financeiros de redução dos gastos públicos. Como consequência, o Ministério da Saúde procura a reestruturação do sector como um marco de desenvolvimento dos processos de reforma do Estado.

O empenhamento organizacional por si só não garante o sucesso organizacional, mas poderá ser uma mais valia quando os colaboradores se comprometem com os objetivos da organização e se envolvem com o seu sucesso, contribuindo para uma oferta de serviços mais humanizados e de melhor qualidade.

Relativamente ao empenhamento profissional o mesmo deve ser entendido como um construto multidimensional e que tem consequências mais acentuadas no desempenho profissional que o empenhamento organizacional.

Quanto ao conflito é aqui entendido como um fenómeno inevitável na vida organizacional: surge nas relações entre indivíduos de um mesmo grupo, entre grupos, entre os diferentes níveis organizacionais e entre organizações. A complexidade de uma organização de saúde onde se interligam diferentes competências, conhecimentos, capacidades e atividades, de cuja adequada coordenação depende o alcance dos objetivos estabelecidos, bem como a interação e a interdependência entre os diferentes elementos que fazem parte da organização, geram tendências para a ação, constituindo-se como uma fonte potencial de conflito (Deutsch, 2003).

O interesse pelo estudo do empenhamento organizacional tem, também, justificação pela invocação da relação causa-efeito entre o empenhamento dos indivíduos e a competitividade das organizações.

Rego (2003) afirma que esta lógica subjacente é simples: inseridas em ambientes turbulentos e competitivos, as organizações de colaboradores que adotem comportamentos espontâneos de respostas a problemas, inovadores e criativos, que se identifiquem com a organização e que atuem como bons cidadãos organizacionais, tornam-se mais competitivas.

Vários estudos de diferentes autores reforçam o empenhamento organizacional como o ponto em que o individuo aceita e interioriza os objetivos e valores de uma organização e como vê a função organizacional em termos da sua contribuição para esses objetivos e valores, para além de quaisquer instrumentalismos pessoais que possam reforçar o seu contributo (DeCottis e Summers, 1987). 
A importância de compreender o conceito de empenhamento organizacional deve-se à evidência de que ele é responsável pelas atitudes e comportamentos, como a assiduidade, a pontualidade, a intenção de abandonar a organização, as atitudes face à mudança, o desempenho do colaborador, os comportamentos de cidadania organizacional, os comportamentos de negligência e de abandono e o desempenho da organização (Allen e Meyer, 1996; Meyer, 1997; Allen e Meyer, 2000; Meyer e Herscovitch, 2001; Rego e Souto, 2002).

Allen e Meyer (1991) revelam a ideia de que o empenhamento organizacional é um estado psicológico que caracteriza o relacionamento do colaborador com a organização e tem implicações na decisão de continuar membro da organização. Os fundamentos consensuais entre os investigadores, para a definição de empenhamento organizacional, são: o desejo de permanecer membro da organização; o orgulho por pertencer à organização; a identificação com os objetivos, metas e valores da organização; o envolvimento, o esforço e o empenho exercido a favor da organização ${ }^{(10)}$.

A partir da década de noventa tornou-se um consenso geral o facto de que o empenhamento organizacional é um constructo multidimensional. A partir da análise das diversas definições sobre o tema, Meyer e Allen (1991) notaram que estas refletiam três grandes áreas: Affective commitment (empenhamento afetivo) - referindo-se a um envolvimento emocional do empregado em que ocorre uma identificação com a organização: empregados com um forte comprometimento afetivo permanecem na organização porque assim o querem; Continuance commitment (empenhamento instrumental) - referindo-se a um comprometimento percebido como custos associados por deixar a organização: empregados cujo vínculo principal com a organização é baseado no comprometimento instrumental, permanecem na organização porque precisam; Normative commitment (empenhamento normativo) refletindo um sentimento de obrigação de permanecer na organização: empregados com um alto nível de comprometimento normativo sentem que devem permanecer na organização.

Num outro contexto, a falta de consenso na definição e medição do empenhamento na profissão é bem percetível na análise da literatura sobre este tema e decorre do facto de cada autor avançar com uma concetualização própria, sem a preocupação de integrar conceitos já existentes. A falta de clarificação deste conceito tem levado a que vários termos sejam usados com o mesmo objetivo de identificar a importância da ocupação na vida do indivíduo, nomeadamente o empenhamento na ocupação, o empenhamento na carreira e o empenhamento na profissão. 
Apesar da discussão teórica, há um ponto consensual entre as diferentes abordagens: o empenhamento profissional ou o empenhamento na carreira, reflete a ligação psicológica do indivíduo em relação à profissão e/ou carreira. Aranya e Ferris (1983) e Morrow e Wirth (1989) defendem o empenhamento profissional como a intensidade relativa da identificação e envolvimento numa profissão. Para estes autores o empenhamento profissional pressupõe: a crença e aceitação dos objetivos e valores de uma profissão; a prontidão em exercer um esforço em prol dela e o desejo de permanecer na profissão. Já Blau et al (1993), referem que o empenhamento profissional envolve a atitude, incluindo afeto, crença e intenção comportamental, em relação à profissão.

Meyer, Allen e Smith (1993) e Irving et al (1997), discordam deste conceito por considerarem que na sua definição Blau et al (1993) encaram o empenhamento como um construto unidimensional, como uma ligação afetiva em relação à profissão. Estes autores consideram ainda o empenhamento profissional constituído por três dimensões: a dimensão afetiva, a dimensão instrumental e a dimensão normativa. $\mathrm{O}$ empenhamento profissional afetivo refere-se à identificação e envolvimento nos objetivos da profissão, assim como ao afeto em relação à profissão. $\mathrm{O}$ empenhamento profissional instrumental diz respeito aos investimentos feitos na profissão e que seriam perdidos caso a abandonássemos bem como à perceção de ausência de alternativas em termos de profissão. $\mathrm{O}$ empenhamento profissional normativo centra-se, então, no dever e responsabilidade sentidos e que levam o indivíduo a permanecer na profissão escolhida.

No que diz respeito ao conflito organizacional, os diversos autores focalizam a definição de conflito em fatores psicológicos, como sejam a divergência de interesses, ideias ou desejos, que são percebidos pelos intervenientes no conflito, mas que não são alcançados por ambos em simultâneo.

Os conflitos são essencialmente um fenómeno relacional que provoca um sentimento de ausência de equilíbrio ou harmonia, que se desenvolve dentro de um indivíduo (individual), entre duas ou mais pessoas (interpessoal), dentro de um grupo (intragrupal), ou entre dois ou mais grupos (intergrupal) (Nursing, 2000).

Jaca e Riquelme (1998) afirmam que os conflitos nas organizações, não devem ter um nível extremamente baixo, nem um nível extremamente elevado de conflito para produzir bons resultados. Segundo os autores, só uma taxa de conflito moderada poderá levar a resultados positivos.

Rahim (2002) refere que o que é preciso para as organizações contemporâneas é a gestão de conflitos e não a resolução de conflitos. A 
gestão de conflitos não implica necessariamente a evasão, redução ou cessação dos conflitos. Trata-se de conceber estratégias eficazes a nível macro, para minimizar as disfunções de conflito e de reforçar as funções construtivas do conflito, a fim de melhorar a aprendizagem e eficácia em uma organização.

Adequadamente geridos, os conflitos, podem ser uma força criativa para o indivíduo e para a organização, pois as diferenças de opinião são valiosas fontes de fertilização cruzada (Penley e Gould, 1988). Por outras palavras, o conflito apresenta possibilidades interessantes sobre o futuro (se for gerido de forma positiva, construtiva), porque as situações difíceis e os relacionamentos são considerados como o que faz, verdadeiramente, as pessoas crescer e reforçar as suas relações, mais do que aumentar os custos (Chen e Tjosvold, 2002).

Assim, as organizações, têm cada vez mais, movido esforços, não para eliminar o conflito, mas para aumentar a gestão eficaz de conflitos, reconhecendo que o conflito é apenas um subproduto esperado (e às vezes até desejável) dos processos organizacionais (Higinite et al, 2002).

As estratégias de gestão de conflitos podem ser definidas como o conjunto de respostas dado pelos envolvidos perante a emergência de ideias, opiniões e/ou objetivos divergentes( Dechurch e Marks, 2001; Pruitt e Rubin, 1986).

Vários modelos com diferentes estilos de lidar com o conflito organizacional foram propostos ao longo dos anos por teóricos organizacionais. O modelo de cinco estilos de gestão de conflitos foi primeiramente concebido por Blake e Mouton em 1964, Thomas, 1992; Blake e Mouton, 1997 (in Rahim, 2001), e foi baseado em duas dimensões: preocupação com a produção e preocupação com as pessoas.

As dimensões propostas por Rahim (2001) são "interesses próprios" e "interesses dos outros". A combinação destas dimensões produz cinco estilos de administração de conflitos. 


\section{Figura I}

Modelo Bidimensional de Estilos de Gestão de Conflitos (Adaptado de Brahnam et al, 2005)

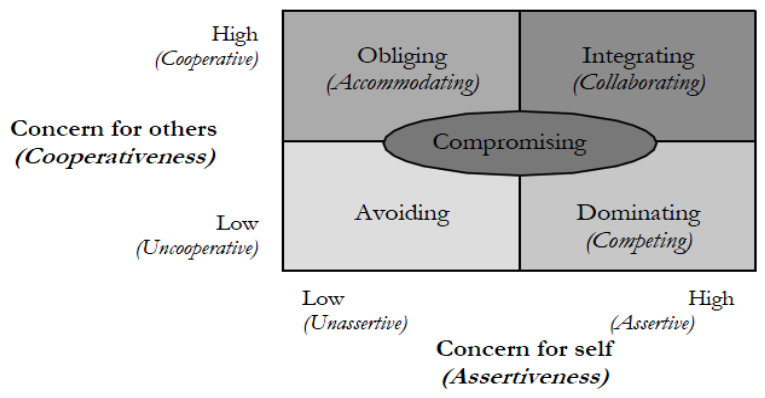

De acordo com Rahim (2001) as características dos cinco estilos de gestão de conflitos podem ser resumidas da seguinte forma:

O estilo Dominação - corresponde a uma grande preocupação com interesses próprios e baixa preocupação com interesses dos outros, onde uma das partes procura satisfazer seus interesses à custa da outra parte. Trata-se de uma personalidade assertiva e não cooperativa porque uma das partes em conflito é agressiva e tenta satisfazer os seus interesses, independentemente do impacto sobre a outra parte envolvida no conflito. Uma pessoa dominadora ou competitiva vai ao limite a fim de alcançar seus objetivos e, como resultado, ignora as expectativas ou necessidades das outras partes envolvidas.

O estilo Integração - predominante quando ambas as partes em conflito desejam satisfazer totalmente os interesses de todas as partes. Este estilo corresponde a uma grande preocupação com interesses próprios e dos outros. Trata-se de uma personalidade assertiva e cooperativa resultando em "ganha-ganha", em que cada parte envolvida no conflito deseja satisfazer plenamente as necessidades de todas as partes envolvidas, trabalhando em conjunto, trocando informações para encontrar soluções aceitáveis para ambas as partes.

O estilo Compromisso - marcado por ações de "dar e receber", onde ambas as partes abrem mão de algo com o intuito de encontrar um resultado aceitável para ambos. Este estilo corresponde a uma moderada preocupação com interesses próprios e dos outros. Trata-se de uma personalidade assertiva e cooperativa média, em que se busca uma solução de meio termo e onde ambas as partes têm "algo para dar" e "algo para receber", procurando-se soluções mutuamente aceitáveis.

O estilo Evitação - associado com a retirada, ignorância ou desatenção face à situação conflituante. Uma pessoa que evita o conflito 
falha em satisfazer seus próprios pontos de vista, bem como os da outra parte envolvida. Trata-se de uma personalidade não assertiva e não cooperativa, em que o desfecho da situação conflitual é normalmente "perde-perde", porque ambas as partes se abstêm de comunicar as suas necessidades e consequentemente não são atendidas.

O estilo Acomodação - associado a minimizar as diferenças, ao mesmo tempo que se maximizam os fatores comuns, para que parte oposta seja satisfeita. A pessoa acomodada negligencia o seu próprio ponto de vista em favor da outra parte. Trata-se de uma personalidade assertiva e não cooperativa, em que a solução do conflito resulta em "perde-ganha", traduzindo-se por autossacrifício dos próprios interesses para satisfazer os interesses do outro, desenvolvendo atitudes de aceitação da vontade do adversário. Os indivíduos procuram o consentimento e aprovação do outro, manifestando ansiedade para ser útil e conseguir a aprovação e apoio dos outros. Estes indivíduos obedecem às ordens de outras pessoas mesmo quando preferiam não o fazer.

Entre os cinco estilos de resolução de conflitos descritos, a literatura parecem favorecer o uso de estilo colaborativo/integrativo e recorda que as estratégias de gestão colaborativa/integrativas geram maior qualidade decisões do que as estratégias de distribuição (Thomas, 1977; Filley, 1978; Jones e White, 1985; Bettenhausen, 1991; Lovelace et al, 2001; Brahnam et al, 2005).

Em suma, a estratégia de gestão de conflitos adotada deve ter como objetivo manter níveis razoáveis de conflito, de forma a estimular processos organizacionais como a criatividade e aprendizagem organizacional (Robbins, 2002). No entanto não existe um estilo definido pelos investigadores que seja amplamente eficaz, pois numa situação conflitual concreta uma estratégia que pode mostrar-se eficaz, noutra situação pode tornar-se altamente ineficaz. Rahim (2001), defende que a um determinado nível ótimo de conflito, corresponde uma elevada performance de trabalho. No entanto, alerta para necessidade de se desenvolver estratégias de gestão de conflitos que se traduzam num aumento da efetividade organizacional. Rahim (2003) menciona também que a gestão adequada dos conflitos, além de se relacionar com a capacidade de contribuir para o desempenho dos grupos e organização, satisfaz concomitantemente as necessidades sociais, morais e éticas dos elementos do grupo ou da organização.

Partindo do enquadramento teórico contextualizado, salientamos que o objetivo principal deste estudo centrou-se na análise do impacto do empenhamento organizacional e profissional dos enfermeiros na adoção de estratégias de resolução de conflitos, em contexto de trabalho. 


\section{MATERIAL E MÉTODOS}

Geograficamente este trabalho foi desenvolvido na Região NUT II Centro e NUT III - Dão-Lafões, tendo recorrido a uma amostra não probabilística e de conveniência, constituída por 102 enfermeiros. A colheita de dados decorreu no período de Maio e Junho de 2011.

$\mathrm{O}$ instrumento de colheita de dados, o qual reúne medidas de avaliação aferidas e validadas para a população portuguesa, era constituído por 5 secções: 1 - Ficha de caracterização sociodemográfica; 2 - Ficha de caracterização profissional dos enfermeiros; 3 - Escala de avaliação do empenhamento organizacional - "Organizational Commitment Questionnaire" (OCQ), de Meyer et al (1993), baseada no Modelo de Três Componentes e que operacionaliza o constructo de empenhamento organizacional, medindo as três dimensões do empenhamento organizacional: afetivo, normativo e instrumental. A adaptação portuguesa do OCQ foi realizada por Bruno (2007), numa escala de respostas tipo Likert de 5 pontos; 4 - Escala de avaliação do empenhamento profissional- "Attitudinal Commitment Scale" (ACS), originalmente desenvolvida por Langford (in Jones, 2000) e adaptada por Jones (2000). Esta escala mede as dimensões Entusiasmo, Satisfação, Interesse, Desafio, Recompensa, Relevância da Enfermagem como profissão e Identificação com o papel de Enfermeiro. O instrumento utiliza uma escala de medição de tipo Likert de 5 pontos. A versão utilizada neste estudo foi a adaptada por Santos (2008); e 5 - Escala de avaliação dos estilos de gestão de conflitos "Rahim Organizational Conflict Inventory - II" (ROCI-II), o qual é composto por 28 itens, os quais incorporam três formas, através das quais se pretende medir de que modo os sujeitos lidam com situações conflituais perante superiores (forma A), subordinados (forma B) e colegas (forma C). Para responder a cada um dos 28 itens foi utilizada uma escala Likert com cinco opções de resposta. Tendo em vista a tradução e adaptação do instrumento para o português nas suas três formas - A, B e C - foi feita por Moreira (2003) sendo esta a versão utilizada neste estudo.

Para análise dos dados foi construída uma base de dados no IBM Staticall Package for the Social Sciences (SPSS®, versão 19.0.1).

$\mathrm{O}$ estudo assenta numa metodologia de natureza quantitativa, transversal, seguindo uma via descritivo-correlacional, com recurso ao inquérito por questionário. Para este estudo, foram selecionadas as seguintes variáveis: as estratégias de resolução de conflitos como variável dependente e como variáveis independentes o empenhamento organizacional, o empenhamento profissional. e ainda algumas variáveis 
de contexto sociodemográfico e profissional. Tendo como finalidade obter um conhecimento mais alargado foram ainda consideradas como variáveis atributo o Curso de Especialização em Enfermagem e o rendimento mensal médio.

A articulação entre as variáveis estudadas, tendo em conta a perspetiva de funcionamento da análise inferencial, é representada de forma esquematizada no modelo conceptual da figura II.

Figura II

Modelo conceptual da relação entre as variáveis estudadas na investigação

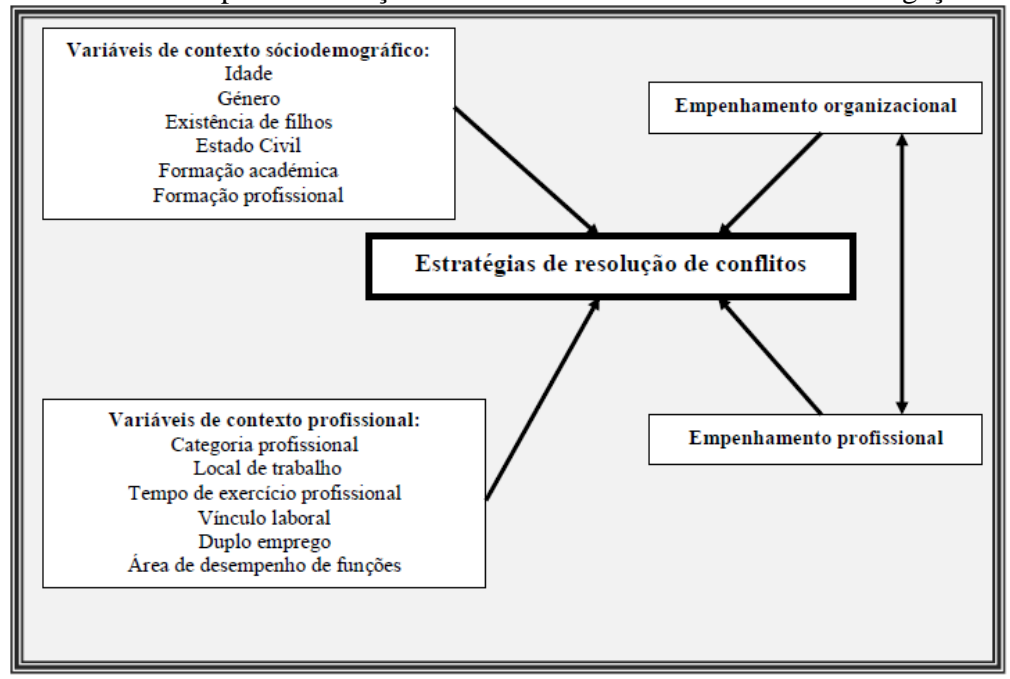

Atendendo ao problema e aos objetivos do estudo (sua operacionalização) e na sequência dos fundamentos teóricos, que suportam a ideia que as estratégias de resolução de conflitos são condicionados por variáveis de contexto pessoal, social e profissional, formularam-se as seguintes hipóteses gerais que pretendem testar, numa amostra de enfermeiros portugueses, o modelo conceptual proposto:

Hipótese 1 - Existem efeitos significativos das variáveis sociodemográficas (idade, género, existência de filhos, estado civil e formação académica) nas estratégias de resolução de conflitos adotadas pelos enfermeiros;

Hipótese 2 - Existem efeitos significativos das variáveis profissionais (formação profissional, categoria profissional, local de trabalho, tempo de exercício profissional, vínculo laboral, duplo emprego e área de 
desempenho de funções) nas estratégias de resolução de conflitos adotadas pelos enfermeiros;

Hipótese 3 - Existe uma associação positiva entre o empenhamento organizacional e o empenhamento profissional dos enfermeiros;

Hipótese 4 - Existe uma associação significativa entre o empenhamento organizacional e as estratégias de resolução de conflitos adotadas pelos enfermeiros;

Hipótese 5 - Existe uma associação significativa entre o empenhamento profissional e as estratégias de resolução de conflitos adotadas pelos enfermeiros.

Hipótese 6 - As variáveis independentes (idade, tempo de serviço na instituição, empenhamento afetivo, empenhamento normativo, empenhamento de continuidade, interesse, relevância e desafio da profissão de enfermagem) predizem significativamente as estratégias de resolução de conflitos adotadas pelos enfermeiros.

\section{APRESENTAÇÃO E ANÁLISE DOS RESULTADOS}

Os resultados do estudo revelam uma amostra constituída por: 102 enfermeiros, sendo 84 do género feminino $(82,4 \%)$ e 18 do género masculino (17,6\%). A média idade é de 39,33 anos $(D p=9.226)$. 70.5\% são casados, $16.7 \%$ são solteiros, $10.8 \%$ são divorciados e $2 \%$ são viúvos. $74.5 \%$ dos enfermeiros têm filhos e destes $63,5 \%$ têm dois ou mais filhos. Quanto ao grau académico e formação profissional: $79,4 \%$ são licenciados, 10,8\% são mestres em Gestão e Administração de Unidades de Saúde, 18,2\% em Família e Sistemas Sociais, 9,1\% em Enfermagem Transcultural, 9,1\% em Educação para a Saúde 9,1\% em Sociopsicologia da Saúde e 9,1\% em Saúde Ocupacional, 7,8\% têm uma pós graduação para além da licenciatura, $62,5 \%$ pós-graduação em Urgência e Emergência, 12,5\% pós-graduação em Família e Sistemas Sociais, 12,5\% em Nutrição Clinica e 12,5\% em Cuidados Continuados e apenas 2\% possuem grau de bacharel.

$\mathrm{Na}$ amostra, 24,48\% dos enfermeiros têm Curso de Especialização em Enfermagem e destes 37,5\% em Saúde Comunitária, 29,2\% em Saúde Infantil e Pediátrica, 16,7\% em Saúde Materna e Obstétrica, 12,5\% em Reabilitação e 4,2\% em Saúde Mental e Psiquiátrica. 81,4\% dos enfermeiros não tem formação específica em gestão e apenas $18,5 \%$ o têm.

Quanto à caracterização profissional concluímos que: $50 \%$ dos enfermeiros são graduados, $28,4 \%$ são enfermeiros, $14,7 \%$ são enfermeiros especialistas e 6,9\% são enfermeiros chefes/coordenadores. 
No que se refere ao tipo de unidade onde exercem funções: $49 \%$ dos enfermeiros exercem funções numa UCSP, 20,6\% numa USF Modelo B, $18,6 \%$ numa USF Modelo A, 6,9\% numa SUB, 2,0\% numa USP, 2,0\% no Conselho Clínico do ACES e apenas 1,0\% numa UCC.

Em relação ao vínculo laboral: $72,5 \%$ possui contrato de trabalho de funções públicas e $27,5 \%$ possui contrato de trabalho a termo certo.

Relativamente à existência de duplo emprego: $82,4 \%$ não trabalha noutro local para além da unidade de saúde, enquanto $17,6 \%$ tem um segundo emprego.

A distribuição, quanto à área de desempenho de funções, verificamos que: $74,6 \%$ exerce funções em cuidados de enfermagem gerais, 7,8\% em cuidados de enfermagem especializados, $7,8 \%$ dos enfermeiros acumulam funções relativas aos cuidados de enfermagem gerais, cuidados de enfermagem especializados e funções de gestão nas respetivas unidades onde trabalham e 5,9\% dos exercem exclusivamente funções de gestão.

O tempo de exercício profissional que oscila entre um mínimo de 3 anos de serviço e um máximo de 36 anos de serviço. O tempo médio de exercício profissional é de 16,55 anos ( $D p=9.394)$.

O rendimento mensal médio liquido varia entre um mínimo de 800 euros e um máximo de 2600 euros mensais e em média é de 1228,45 euros $(D p=346.588)$, verificando-se que os enfermeiros têm um rendimento mensal médio superior ao das enfermeiras.

A caracterização do empenhamento organizacional mostra que 65,6\% dos inquiridos apresentam níveis moderados, 30,5\% apresentam níveis elevados e 3,9\% apresentam níveis baixos. Quanto ao empenhamento profissional podemos concluir que: $60,8 \%$ dos inquiridos apresentam níveis moderados, 39,2\% apresentam níveis elevados e nenhum apresenta níveis baixos.

Relativamente às estratégias de resolução de conflitos (ver Quadro I) podemos verificar que as médias nas estratégias dominação $(\bar{x}=13.77)$ e acomodação $(\bar{x}=19.78)$ são mais elevadas na forma $\mathrm{A}$, o que revela que os enfermeiros adotam mais as estratégias de resolução de conflito dominação e acomodação face ao chefe. A média nas estratégias integração $(\bar{x}=24.47)$ e compromisso $(\bar{x}=13.83)$ são mais baixas na forma $\mathrm{A}$, ou seja, os enfermeiros usam menos as estratégias integrativas e de compromisso quando em situação conflitual com o chefe. A média na estratégia evitação( $(\bar{x}=27.70)$ é mais elevada na forma $\mathrm{B}$, pelo que podemos afirmar que os enfermeiros adotam mais as estratégias de evitação no confronto com os subordinados. A média nas estratégias integração $(\bar{x}=28.44)$ e compromisso $(\bar{x}=14.22)$ são mais elevadas na forma $\mathrm{C}$, ou seja os enfermeiros adotam mais as estratégias integrativas e 
de compromisso quando a situação conflitual surge com os colegas. A média na estratégia dominação $(\bar{x}=12.97)$ é mais é baixa na forma $\mathrm{C}$, ou seja os enfermeiros adotam menos a estratégia dominação quando a situação conflitual surge com os colegas.

\section{Quadro I}

Média das estratégias de resolução de conflitos: Forma A - face ao Chefe ( $s$ ) /superior (es) hierárquico (s), Forma B - face aos Subordinado (s) e Forma C-face aos Colega $(s)$

\begin{tabular}{|l|c|c|c|}
\hline Estratégia & $\begin{array}{c}\text { Média A } \\
\text { (Chefe) }\end{array}$ & $\begin{array}{c}\text { Média B } \\
\text { (Subordinado) }\end{array}$ & $\begin{array}{c}\text { Média C } \\
\text { (Colega) }\end{array}$ \\
\hline Integração & 24,47 & 27,70 & 28,44 \\
\hline Evitação & 18,51 & 27,70 & 19,20 \\
\hline Dominação & 13,77 & 13,17 & 12,97 \\
\hline Acomodação & 19,78 & 19,01 & 19,01 \\
\hline Compromisso & 13,83 & 14,01 & 14,22 \\
\hline
\end{tabular}

O estudo de associação entre as variáveis permite-nos concluir que existem efeitos significativos das variáveis sociodemográficas (género, grupo etário, formação académica e formação em gestão) na adoção de algumas estratégias de resolução de conflitos adotadas pelos enfermeiros, pelo que aceitamos parcialmente a hipótese 1 .

Os resultados mostram que:

Género - os enfermeiros usam mais a estratégia dominação - face aos subordinados $(\mathrm{t}=2,136, \mathrm{p}=, 035)$ que as enfermeiras;

Grupo etário - os enfermeiros mais jovens adotam mais a estratégia evitação face ao chefe $(\mathrm{F}=3,501 ; \mathrm{p}=, 034)$, que os enfermeiros com idades mais avançadas;

Formação académica - os enfermeiros com grau académico de bacharel adotam mais frequentemente a estratégia - dominação face ao chefe $(\mathrm{F}=2,519 ; \mathrm{p}=, 049)$, que os enfermeiros com grau académico de mestre;

Formação em gestão - os enfermeiros com formação em gestão adotam de forma mais continuada a estratégia - dominação face ao chefe $(\mathrm{t}=2,424, \mathrm{p}=, 020)$ e subordinados $(\mathrm{t}=2,166, \mathrm{p}=, 033)$.

Em relação às variáveis profissionais (categoria profissional, local de trabalho, tempo de exercício profissional e área de desempenho de funções) verificamos que influenciam as estratégias de resolução de 
conflitos adotadas pelos enfermeiros pelo que aceitamos parcialmente a hipótese 2.

As estratégias de resolução de conflitos adotadas são influenciadas de forma estatisticamente significativa pelas variáveis profissionais e os resultados revelam que: os enfermeiros com a categoria de enfermeiro e enfermeiro graduado adotam de forma mais acentuada a estratégia evitação face ao chefe $(\mathrm{F}=4,649 ; \mathrm{p}=, 004)$ e aos colegas $(\mathrm{F}=6,649$; $\mathrm{p}=, 000)$; a adoção da estratégia - dominação face ao chefe $(\mathrm{F}=5,427$; $\mathrm{p}=, 006)$, subordinados $(\mathrm{F}=4,542 ; \mathrm{p}=, 013)$ e colegas $(\mathrm{F}=6,573 ; \mathrm{p}=, 002)$ é influenciada local onde trabalha; os enfermeiros que têm entre 5 e 15 anos de serviço adotam mais a - evitação face ao chefe $(\mathrm{F}=2,834$; $\mathrm{p}=, 042)$ e aos colegas $(\mathrm{F}=3,079 ; \mathrm{p}=, 031)$; os enfermeiros que exercem funções nos cuidados gerais adotam mais a estratégia - evitação face ao chefe $(\mathrm{F}=3,267 ; \mathrm{p}=, 031)$ e aos colegas $(\mathrm{F}=7,242 ; \mathrm{p}=, 001)$ que os enfermeiros que exercem exclusivamente funções de gestão.

Tendo em conta os resultados obtidos pelas correlações (Quadro II), referentes as três dimensões e os score total do empenhamento organizacional e as três dimensões e o score total do empenhamento profissional constatamos a existência de associações estatisticamente significativas entre estas variáveis. Em concreto, constatamos que:

$\mathrm{O}$ empenhamento afetivo revela uma associação positiva baixa com as dimensões (interesse: $\mathrm{r}=, 221 ; p=, 026$; relevância: $\mathrm{r}=, 206 ; p=, 038$; desafio: $\mathrm{r}=, 259 ; p=, 009)$ e score total do empenhamento profissional $(\mathrm{r}=, 276 ; p=, 005)$, o que indicia que quanto maior é o empenhamento afetivo maior é o empenhamento profissional no seu global.

$\mathrm{O}$ empenhamento normativo apresenta uma correlação positiva baixa com a dimensão interesse $(\mathrm{r}=, 284 ; p=, 004)$ da enfermagem como profissão e o score total do empenhamento profissional $(\mathrm{r}=, 211 ; p=, 033)$, ou seja, os enfermeiros que se sentem obrigados a permanecer na organização que revelam maior interesse na profissão e um nível mais elevado de empenhamento profissional.

O empenhamento organizacional total apenas está correlacionado positivamente, de forma baixa, com a dimensão interesse $(\mathrm{r}=, 248$; $p=, 012)$ e de forma muito baixa com o score total do empenhamento profissional $(\mathrm{r}=, 094 ; p=, 042)$ o que nos permite afirmar que quanto maior o nível de empenhamento organizacional maior o nível de empenhamento profissional. 


\section{Quadro II}

Resultados da correlação de Pearson entre o empenhamento organizacional e o empenhamento profissional dos enfermeiros

\begin{tabular}{|c|c|c|c|c|c|}
\hline Empenhamento organizacional & & & & & Emp. \\
\hline Empenhamento profissional & & Afectivo & Normativo & Continuidade & Total \\
\hline \multirow[b]{2}{*}{ Interesse } & $\mathrm{r}$ & .221 & .284 & .067 & .248 \\
\hline & $p$ & .026 & .004 & .503 & .012 \\
\hline \multirow[b]{2}{*}{ Relevância } & $\mathrm{r}$ & .206 & .140 & .008 & .137 \\
\hline & $p$ & .038 & .159 & .933 & .171 \\
\hline \multirow[b]{2}{*}{ Desafio } & $\mathrm{r}$ & .259 & .069 & -.061 & .348 \\
\hline & $p$ & .009 & .490 & .542 & .202 \\
\hline \multirow[b]{2}{*}{$\begin{array}{l}\text { Empenhamento Profissional } \\
\text { Total }\end{array}$} & $\mathrm{r}$ & .276 & .211 & .005 & .094 \\
\hline & $p$ & .005 & .033 & .958 & .042 \\
\hline
\end{tabular}

Considerando os resultados obtidos pelas correlações (Quadro III), tendo por referência as três dimensões e os score total do empenhamento organizacional e as estratégias de resolução de conflitos face ao chefe, subordinados e colegas, constatamos a existência de associações estatisticamente significativas entre estas variáveis. Analisando os dados verificamos que:

O empenhamento afetivo apenas revela uma associação positiva baixa com a estratégia - compromisso face ao chefe $(\mathrm{r}=, 238 ; p=, 016)$, ou seja, quanto maior é o nível de empenhamento afetivo maior é o compromisso enquanto estratégia de resolução de conflitos face ao chefe.

$\mathrm{O}$ empenhamento normativo revela uma associação positiva baixa com a estratégia - compromisso face ao chefe $(\mathrm{r}=, 218 ; p=, 028)$ e a estratégia - evitação face aos colegas $(\mathrm{r}=, 211 ; p=, 033)$, pelo que podemos deduzir que quanto maior é o nível de empenhamento normativo mais utilizam a estratégia compromisso face ao chefe.

$\mathrm{O}$ empenhamento de continuidade tem uma correlação positiva baixa com as estratégias de resolução de conflitos - acomodação face aos subordinados $(\mathrm{r}=, 230 ; p=, 020)$ e colegas $(\mathrm{r}=, 230 ; p=, 020)$ e uma correlação positiva baixa com a estratégia de resolução de conflitos evitação face aos colegas $(\mathrm{r}=, 316 ; p=, 001)$, ou seja, quanto maior é o nível de empenhamento continuidade mais utilizam a estratégia acomodação na resolução de conflitos face aos subordinados e colegas e maior é a utilização da evitação nas estratégias de resolução de conflitos face aos colegas.

O empenhamento organizacional total está positivamente correlacionado de forma baixa com as estratégias de resolução de conflitos - compromisso face ao chefe $(\mathrm{r}=, 252 ; p=, 011)$ e a estratégia - 
evitação face aos colegas $(\mathrm{r}=, 231 ; p=, 019) \quad e$ positivamente correlacionado de forma muito baixa com a estratégia - dominação face aos subordinados $(\mathrm{r}=, 196 ; p=, 049)$ e pelo que podemos afirmar que quanto maior é o nível de empenhamento organizacional mais usam as estratégias de resolução de conflitos - compromisso face ao chefe, a estratégia - dominação face aos subordinados e a estratégia - evitação face aos colegas.

\section{Quadro III}

Resultados da correlação de Pearson entre o empenhamento organizacional e as estratégias de resolução de conflitos face ao chefe/superior hierárquico - Forma A, subordinados - Forma B e colegas - Forma C

\begin{tabular}{|c|c|c|c|c|c|c|c|c|c|c|c|c|c|c|c|c|}
\hline \multirow[b]{3}{*}{$\begin{array}{l}\text { Empenhamento } \\
\text { organizacional }\end{array}$} & & \multicolumn{15}{|c|}{ Estratégias de resolução de conflitos } \\
\hline & & \multicolumn{5}{|c|}{$\begin{array}{c}\text { Forma A } \\
\text { Chefes }\end{array}$} & \multicolumn{5}{|c|}{$\begin{array}{c}\text { Forma B } \\
\text { Subordinados }\end{array}$} & \multicolumn{5}{|c|}{$\begin{array}{l}\text { Forma C } \\
\text { Colegas }\end{array}$} \\
\hline & & 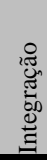 & 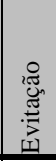 & : & 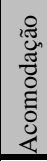 & 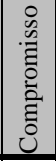 & 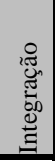 & 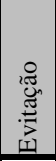 & 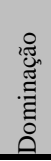 & 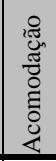 & 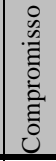 & 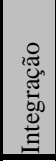 & 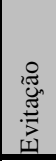 & 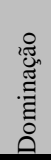 & 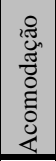 & 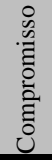 \\
\hline \multirow[b]{2}{*}{$\begin{array}{l}\text { Empenhamento } \\
\text { afectivo }\end{array}$} & $\mathrm{r}$ & .139 & -.032 & .146 & .081 & .238 & .146 & .146 & .194 & .129 & .052 & .193 & .027 & .140 & .129 & .181 \\
\hline & $p$ & .163 & .748 & .144 & .421 & .016 & .144 & .144 & .051 & .196 & .603 & .052 & .791 & .159 & .196 & .068 \\
\hline \multirow{2}{*}{$\begin{array}{l}\text { Empenhamento } \\
\text { normativo }\end{array}$} & $\mathrm{r}$ & .157 & .133 & .007 & .093 & .218 & .124 & .124 & .140 & .075 & .085 & .115 & .211 & .157 & .075 & .133 \\
\hline & $p$ & .115 & .182 & .441 & .351 & .028 & .215 & .215 & .159 & .452 & .395 & .249 & .033 & .115 & .452 & .182 \\
\hline \multirow{2}{*}{$\begin{array}{l}\text { Empenhamento } \\
\text { de continuidade }\end{array}$} & $\mathrm{r}$ & .065 & .148 & .120 & .129 & .181 & -.020 & -.020 & .184 & .230 & -.056 & .005 & .316 & .117 & .230 & .070 \\
\hline & $p$ & .517 & .137 & .230 & .197 & .069 & .845 & .845 & .064 & .020 & .576 & .960 & .001 & .242 & .020 & .486 \\
\hline \multirow{2}{*}{$\begin{array}{l}\text { Empenhamento } \\
\text { organizacional } \\
\text { total }\end{array}$} & $\mathrm{r}$ & .151 & .115 & .125 & .118 & .252 & .106 & .106 & .196 & .155 & .045 & .123 & .231 & .168 & .155 & .151 \\
\hline & $p$ & .130 & .249 & .211 & .236 & .011 & .289 & .289 & .049 & .120 & .651 & .219 & .019 & .091 & .120 & .130 \\
\hline
\end{tabular}

Considerando os resultados obtidos pelas correlações (Quadro IV), tendo por referência as três dimensões e os score total do empenhamento profissional e as estratégias de resolução de conflitos face ao chefe, subordinados e colegas, constatamos a existência de associações estatisticamente significativas entre estas variáveis. Analisando as associações verificamos que:

A dimensão interesse da profissão de enfermagem revela uma associação positiva moderada com a estratégia - integração face ao chefe $(\mathrm{r}=, 408 ; p=, 000)$, subordinados $(\mathrm{r}=, 431 ; p=, 000)$ e colegas $(\mathrm{r}=, 448 ; p=, 000) \quad$ e uma associação positiva baixa com a estratégia acomodação face ao chefe $(\mathrm{r}=, 278 ; p=, 003)$ e com a estratégia compromisso face aos subordinados $(\mathrm{r}=, 247 ; p=, 012)$ e colegas $(\mathrm{r}=, 318 ; p=, 001)$. 
A dimensão relevância da profissão de enfermagem revela uma associação positiva baixa com a estratégia - integração face ao chefe $(\mathrm{r}=, 312 ; p=, 001)$, subordinados $(\mathrm{r}=, 320 ; p=, 001)$ e colega $(\mathrm{r}=, 288$; $p=, 003) \quad s$, pelo que podemos deduzir que quanto maior é o nível de relevância mais utilizam a estratégia - integração face ao chefe, subordinados e colegas.

A dimensão desafio da profissão de enfermagem revela uma associação positiva baixa com a estratégia - integração face ao chefe $(\mathrm{r}=, 336 ; p=, 001)$ e compromisso face aos colegas $(\mathrm{r}=, 280 ; p=, 004)$, uma associação moderada com a estratégia - integração face aos subordinados $(\mathrm{r}=, 505 ; p=, 000)$ e colegas $(\mathrm{r}=, 408 ; p=, 000)$ e uma associação positiva muito baixa com a estratégia - compromisso face ao chefe $(\mathrm{r}=, 197 ; p=, 047)$.

O empenhamento profissional total está positivamente correlacionado de forma moderada com a estratégia - integração face ao chefe $(\mathrm{r}=, 432$; $p=, 000)$, colegas $(\mathrm{r}=, 500 ; p=, 000)$ e subordinados $(\mathrm{r}=, 461 ; p=, 000)$ e apresenta uma correlação baixa com a estratégia-compromisso face aos subordinados $(\mathrm{r}=, 228 ; p=, 021)$ e colegas $(\mathrm{r}=, 262 ; p=, 008)$. Perante estas análises podemos concluir que os enfermeiros mais empenhados profissionalmente demonstram que utilizam mais as estratégias integrativas e de compromisso na resolução de conflitos quer seja face ao chefe, aos subordinados ou aos colegas.

O estudo das variáveis independentes na predição da variável dependente foi realizado através de modelos de regressão linear múltipla hierárquica, com recurso ao método stepwise. Efectuámos Análises de Regressão Múltipla Passo a Passo, em que testamos, de forma independente, cada uma das dimensões/factores da Escala de Resolução de Conflitos foram utilizadas como variáveis dependentes e as variáveis idade, tempo de serviço na instituição, empenhamento afectivo, empenhamento normativo, empenhamento de continuidade, interesse, relevância e desafio da profissão de enfermagem - como variáveis independentes (ver quadro $\mathrm{V}$ ). 


\section{Quadro IV}

Resultados da correlação de Pearson entre o empenhamento profissional e as estratégias de resolução de conflitos face ao chefe/superior hierárquico - Forma A, subordinados - Forma B e colegas - Forma C

\begin{tabular}{|c|c|c|c|c|c|c|c|c|c|c|c|c|c|c|c|c|}
\hline \multirow[b]{3}{*}{$\begin{array}{l}\text { Empenhamento } \\
\text { profissional }\end{array}$} & & \multicolumn{15}{|c|}{ Estratégias de resolução de conflitos } \\
\hline & & \multicolumn{5}{|c|}{$\begin{array}{c}\text { Forma A } \\
\text { Chefes }\end{array}$} & \multicolumn{5}{|c|}{$\begin{array}{c}\text { Forma B } \\
\text { Subordinados }\end{array}$} & \multicolumn{5}{|c|}{$\begin{array}{c}\text { Forma C } \\
\text { Colegas }\end{array}$} \\
\hline & & 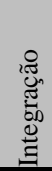 & 忍 & 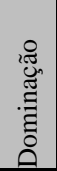 & 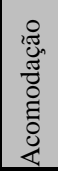 & 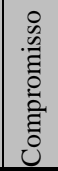 & 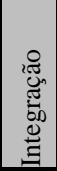 & 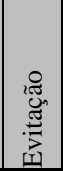 & 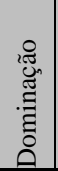 & 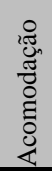 & 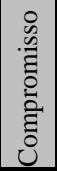 & 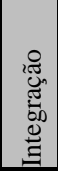 & 莺 & 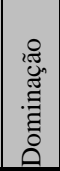 & 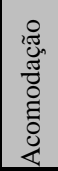 & 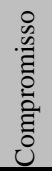 \\
\hline \multirow[b]{2}{*}{ Interesse } & $\mathrm{r}$ & .480 & .134 & -.053 & .287 & .150 & .431 & -.141 & -.141 & .066 & .247 & .448 & .086 & -.097 & .066 & .318 \\
\hline & $p$ & .000 & .178 & .598 & .003 & .132 & .000 & .157 & .157 & .508 & .012 & .000 & .390 & .331 & .508 & .001 \\
\hline \multirow[b]{2}{*}{ Relevância } & $\mathrm{r}$ & .312 & .112 & .024 & .186 & .099 & .320 & \begin{tabular}{|l|}
-.077 \\
\end{tabular} & -.077 & .088 & .056 & .288 & .085 & -.031 & .088 & .071 \\
\hline & $p$ & . 001 & .262 & .807 & .061 & .320 & .001 & .442 & .442 & .377 & .573 & .003 & .397 & .758 & .377 & .447 \\
\hline \multirow[b]{2}{*}{ Desafio } & $\mathrm{r}$ & .336 & -.141 & .026 & -.085 & .197 & .505 & \begin{tabular}{|l|}
-008 \\
\end{tabular} & -.008 & -.053 & .291 & .408 & -.125 & \begin{tabular}{ll|}
-.018 \\
\end{tabular} & -.053 & .280 \\
\hline & $p$ & . 001 & .158 & .772 & .397 & .047 & .000 & .938 & .938 & .596 & .003 & .000 & .210 & .854 & .596 & . 004 \\
\hline \multirow{2}{*}{$\begin{array}{l}\text { Empenhamento } \\
\text { profissional total }\end{array}$} & $\mathrm{r}$ & .432 & .065 & -.002 & .184 & .175 & .500 & \begin{tabular}{|l|}
-.100 \\
\end{tabular} & -.100 & .053 & .228 & .461 & .036 & -.063 & .053 & .262 \\
\hline & $p$ & .000 & .516 & .982 & .064 & .078 & .000 & .319 & .319 & .598 & .021 & .000 & .716 & .532 & .598 & .008 \\
\hline
\end{tabular}

Os resultados permitem-nos aceitar $\mathrm{H}_{6}$, em que as variáveis independentes testadas predizem significativamente as estratégias de resolução de conflitos adotadas pelos enfermeiros da seguinte forma: a dimensão interesse da enfermagem como profissão $\left(R^{2}=, 167 ; F=20,02\right.$; $p=, 000)$ é preditiva na adopção da estratégia de resolução de conflitos integração face ao chefe; as variáveis profissionais, tempo de serviço $\left(\mathrm{R}^{2}=, 154 ; \quad \mathrm{F}=9,057 ; \quad p=, 000\right)$ e as dimensões do empenhamento profissional, interesse $\left(\mathrm{R}^{2}=, 082 ; \mathrm{F}=8,968 ; p=, 003\right)$; e desafio $\left(\mathrm{R}^{2}=, 206\right.$; $\mathrm{F}=8,528 ; p=, 000)$ predizem significativamente o uso da estratégia de resolução de conflitos - acomodação face ao chefe; a dimensão do empenhamento afetivo $\left(\mathrm{R}^{2}=, 238 ; \mathrm{F}=6,02 ; p=, 016\right)$ prediz o uso da estratégia de resolução de conflitos - compromisso face ao chefe; o empenhamento profissional (dimensões desafio - $\mathrm{R}^{2}=, 255 ; \mathrm{F}=34,222$; $p=, 000$ e interesse $\left.-\mathrm{R}^{2}=, 308 ; \mathrm{F}=7,590 ; p=, 007\right)$ prediz significativamente a adopção da estratégia de resolução de conflitos - integração face aos subordinados; o empenhamento profissional (dimensões desafio $\mathrm{R}^{2}=, 255 ; \mathrm{F}=34,222 ; p=, 000$ e interesse $-\mathrm{R}^{2}=, 308 ; \mathrm{F}=7,590 ; p=, 007$ ) 
prediz significativamente a adopção da estratégia de resolução de conflitos - evitação face aos subordinados; o empenhamento organizacional (dimensão empenhamento de continuidade $-\mathrm{R}^{2}=, 053$; $\mathrm{F}=5,60 ; p=, 020)$ prediz de forma significativa a adopção da estratégia de resolução de conflitos - acomodação face aos subordinados; 0 empenhamento profissional (dimensão desafio - $\mathrm{R}^{2}=, 084 ; \mathrm{F}=9,225$; $p=, 003)$ prediz significativamente a adopção da estratégia de resolução de conflitos - compromisso face aos subordinados; o empenhamento profissional (dimensões interesse - $\mathrm{R}^{2}=, 200 ; \mathrm{F}=25,05 ; p=, 000$ e desafio $\left.\mathrm{R}^{2}=, 254 ; \mathrm{F}=7,184 ; p=, 047\right)$ prediz a adopção da estratégia de resolução de conflitos - integração face aos colegas e a idade prediz de forma significativa a adopção da estratégia de resolução de conflitos integração face aos colegas; o empenhamento organizacional (nas três dimensões: empenhamento de continuidade $-\mathrm{R}^{2}=, 100 ; \mathrm{F}=11,082 ; p=, 001$, afetivo $-\mathrm{R}^{2}=, 169 ; \mathrm{F}=3,947 ; p=, 050$ e normativo $-\mathrm{R}^{2}=, 246 ; \mathrm{F}=9,978$; $p=, 002)$ prediz a adopção estratégia de resolução de conflitos - evitação face aos colegas; a idade $\left(\mathrm{R}^{2}=, 100 ; \mathrm{F}=11,082 ; p=, 001\right)$ prediz de forma significativa a adopção da estratégia de resolução de conflitos - evitação face aos colegas; o empenhamento organizacional (dimensão empenhamento de continuidade $\left.-\mathrm{R}^{2}=, 053 ; \mathrm{F}=5,598 ; p=, 020\right)$ prediz de forma significativa a adopção da estratégia de resolução de conflitos acomodação face aos colegas; o empenhamento profissional (dimensão interesse - $\left.\mathrm{R}^{2}=, 101 ; \mathrm{F}=11,28 ; p=, 001\right)$ prediz significativamente a adopção da estratégia de resolução de conflitos - compromisso face aos colegas. 


\section{Paula Pinho e Carlos Albuquerque}

\section{Quadro V}

Regressão linear entre as variáveis - idade, tempo de serviço na instituição, empenhamento afetivo, empenhamento normativo, empenhamento de continuidade,

interesse, relevância e desafio da profissão de enfermagem e as estratégias de resolução de conflitos face ao chefe/superior hierárquico - Forma A, subordinados -

Forma B e colegas - Forma C

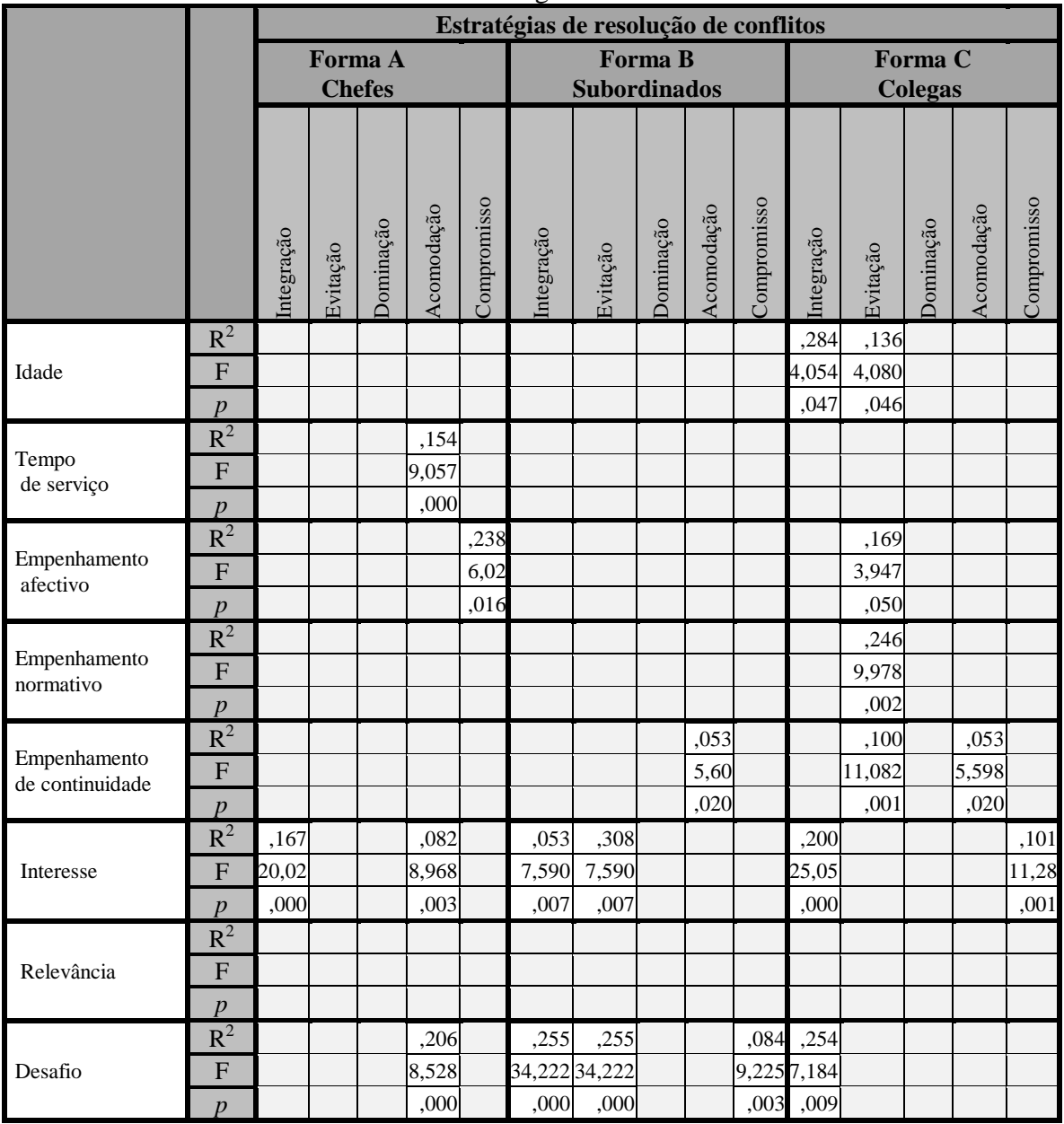

\section{DISCUSSÃO E CONCLUSÕES}

Os enfermeiros demonstram um moderado/alto empenhamento organizacional sendo mais elevado o empenhamento afectivo que o empenhamento normativo ou o empenhamento instrumental. Estes resultados convergem com a evidência empírica de Penley e Gould 
(1988) nos seus estudos efectuados a enfermeiras, no predomínio do empenho afectivo à organização relativamente às outras dimensões. Estudos de Meyer e Allen (1997) são também concordantes no destaque do empenhamento afectivo, seguindo-se o empenhamento normativo e instrumental.

Os enfermeiros demonstram um moderado/alto empenhamento profissional, sendo os resultados revelam que os enfermeiros apresentam valores mais elevados nas dimensões interesse e desafio do que na dimensão relevância da enfermagem como profissão. Estes resultados poderão estar relacionados com o facto de considerarem a enfermagem como uma profissão indispensável, em que se identificam com os valores e objetivos da profissão, que se sentem profissionalmente realizados mas que não terá o reconhecimento social que julgam devido. $\mathrm{O}$ interesse $\mathrm{e}$ desafio da enfermagem como profissão que é percecionado pelos enfermeiros poderão resultar da identificação dos profissionais com os valores e objetivos da enfermagem, da vontade e desejo de exercer a profissão, da intenção de esforço pela mesma (Morrow e Wirth, 1989), da possibilidade de desenvolvimento de competências (Allen e Meyer, 1993), do desenvolvimento de tarefas complexas e desafiantes (Blau, 1999), da autonomia e responsabilidade das funções, da perspetiva de desenvolvimento da profissão no âmbito da formação, gestão e investigação (Colliére, 1999).

A moderada relevância da enfermagem como profissão sentida pelos enfermeiros pode estar relacionada com desvalorização do seu papel como elemento social, a que não é atribuído valor ao trabalho e a falta de relevância social poderá ser devida ao carácter de invisibilidade associado à profissão em virtude dos enfermeiros ainda não terem afirmado os seus efeitos socioeconómicos no trabalho (Colliére, 1999). A falta de reconhecimento social sentido pelos enfermeiros poderá por em causa a imagem social percecionada, o que não significa a verdadeira relevância social da profissão de enfermagem (Colliére, 1999).

No que concerne às estratégias de resolução de conflitos os enfermeiros adotam mais as estratégias de resolução de conflito dominação e acomodação quando em situação conflitual com o chefe e menos as estratégias integrativas e de compromisso. Face aos subordinados os enfermeiros adotam mais as estratégias de evitação perante situações conflituais. Quando a situação conflitual surge com os colegas os enfermeiros adotam mais as estratégias integrativas e de compromisso e menos a estratégia dominação. Tal como nos estudos de Rahim (1986) e de McIntyre (1991), foram percebidas diferenças significativas nos estilos da gestão de conflito utilizados quer pelos 
chefes, pelos subordinados ou pelos colegas, extraindo-se a conclusão que o relacionamento hierárquico condicionava o estilo utilizado pela pessoa envolvida no conflito.

Podemos afirmar que o empenhamento organizacional influencia a adoção de estratégias de resolução de conflitos conforme a situação conflitual surge com o chefe, com os subordinados ou com os colegas, ou seja o papel de uma pessoa enquanto chefe, colega ou subordinado poderá condicionar o estilo escolhido para lidar com o conflito.

Quanto maior o nível de empenhamento organizacional maior o nível de empenhamento profissional. Os resultados podem estar relacionados com a perceção dos enfermeiros de que os objetivos e valores da enfermagem tendem a ser congruentes com os objetivos e valores organizacionais, bem como a proximidade entre a identificação profissional e a organizacional e os papéis profissionais e organizacionais.

Perante estas análises podemos concluir que os enfermeiros mais empenhados profissionalmente demonstram que utilizam mais as estratégias integrativas e de compromisso na resolução de conflitos quer seja face ao chefe, aos subordinados ou aos colegas.

Consideramos que o vínculo que o indivíduo desenvolve com a sua organização é sumamente importante. A conciliação dos empenhamentos profissional e organizacional no cotidiano das organizações favorece a consecução dos propósitos e objetivos institucionais em consequência da eficiência e da eficácia apresentada pelas pessoas que atuam. O desempenho das equipes de trabalho e o impacto dos resultados dependerão muito das suas competências específicas, da comunicação e do envolvimento das pessoas na sua organização e profissão. Pensar no empenhamento dos profissionais como uma forma de contribuir para a identificação dos seus papéis, implica na usa motivação, interesse e envolvimento.

É essencial favorecer e estimular o desenvolvimento do empenhamento afetivo nos indivíduos pelas consequências positivas que ele acarreta na organização e na profissão.

Pensamos que é imprescindível que as organizações possam aplicar o estilo mais adequado de gestão para os seus conflitos, que são inevitáveis. Só aprendendo a gerir eficazmente os seus conflitos internos é que as organizações de saúde estarão preparadas para responder à concorrência que vem do exterior.

São vários os autores que fomentam a ideia de que o desempenho organizacional beneficia com a estimulação do conflito sob determinadas 
condições e que a gestão construtiva dos conflitos nas organizações é um elemento essencial para a eficácia organizacional.

\section{BIBLIOGRAFIA}

Allen, N. J. e Meyer, J. P., (1991). A three-component conceptualization of organizational commitment. Human Resource Management Review, 1(1), 61-89.

Allen, N. e Meyer, J., (1996). Affective, continuance, and normative commitment to the organization: an examination of construct validity. Journal of Vocational Behavior, 49, 252-276.

Allen, N. J. e Meyer, J. P. (1993). Organizational commitment: Evidence of career stage effects? Journal of Business Research, 26, 49-61.

Allen, N. J. e Meyer, J. P. (2000). Construct validation in organizational behavior research: The case of organizational commitment. In R. D. Goffin \& E. Helmes (Eds.), Problems and solutions in human assessment: Honoring Douglas N. Jackson at Seventy (pp. 285-314). Norwell, MA: Kluwer.

Aranya, N. e Ferris, K (1983). Organizational - Professional conflict among U.S. and Israeli professional accountants. Journal of Social Psychology, 119, 153-161.

Bettenhausen, K. L. (1991), Five Years of Groups Research: What We Have Learned and What Needs to be Addressed, Journal of Management, 17(2): 345-381

Blau, G. J.et al (1993). On developing a general index of work commitment. Journal of Vocational Behavior, 42, 298-314.

Blau, G. J. (1999). Early-career job factors influencing the professional commitment of medical technologists. Academy of Management Journal, 42, 687-695.

Bruno, A. (2007). Empenhamento organizacional e liderança: Estudo exploratório numa amostra de trabalhadores do sector da distribuição. Dissertação de Mestrado. Universidade de Lisboa.

Chang, J J. et al. (2002). Comprometimento Organizacional: criatividade e inovação. Revista de Administração Mackenzie. São Paulo, ano 3, n.2, p.13-38.

Chen, G., Tjosvold, D. (2002). Conflict management and team effectiveness in China: The mediating role of justice, Asia Pacific Journal of Management, 19(4): 557-572. 
Collière, M-F, (1989). Promover a vida - da prática das mulheres de virtude aos cuidados de enfermagem. Lisboa: Sindicato dos Enfermeiros Portugueses.

DeChurch, L. A., Marks, M. A. (2001), Maximizing the benefits of task conflict: the role of conflict management, International Journal of Conflict Management, 12(1): 4-22

DeCotiis, T. e Summers, T., (1987). A path analysis of a model of the antecedents and consequences of organizational commitment. Human Relations, 40(7), 445-470.

Deutsch, M. (2003). Cooperation and Conflict: a personal perspective on the history of the social psychology study of conflict resolution. In M. West, D. Tjosvold e K. Smith (Eds.), International Handboock of Organizational Teamwork and Cooperative Working. Chichester: John Wiley e Sons, Ltd.

Filley, A. C. (1978). Some Normative Issues in Conflict Management, California Management Review, 21(2): 61-66.

Hignite, M. A.et al. (2002), Assessing the Conflict Resolution Profiles of Emerging Information Systems Professionals, Journal of Information Systems Education, 13(4): 315-324.

Irving, P. G. et al (1997). Further assessments of a threecomponent model of occupational commitment: Generalizability and differences across occupations. Journal of Applied Psychology, 82(3), 444-452.

Jaca, L. M. e Riquelme, J. M. (1998). Conflicto y Negociación. Madrid: Ediciones Pirámide.

Jones, J. (2000). The Impact of Hospital Mergers on Organizational Culture, Organizational Commitment, Professional Commitment, Job Satisfacion, and Intent to Turnover on registered Professional Nurses on Medical-Surgical Hospital Units. Tese de Doutoramento em Filosofia, State University of New York at Buffalo.

Jones, T. E., White, C. S. (1985). Relationships Among Personality, Conflict Resolution Styles, and Task Effectiveness. Group $e$ Organization Studies, 10(2): 152-167

Lovelace, K. et al (2001), Maximizing cross-functional new product teams ${ }^{\text {ee }}$ innovativeness and constraint adherence: a conflict communications perspective, Academy of Management Journal, 44(4): 779-793.

McIntyre, S. E. (1991). Conflict management by male and female managers as reported by self and by male and female subordinates. Unpublished doctoral thesis. Atlanta, GA: Georgia State University. 
Meyer, J. (1997). Organizational commitment. In C. L. Cooper e I. T. Robertson (Eds.), International Review of Industrial and Organizational Psychology, 12, 175-228. Chichester: John Wiley \& Sons.

Meyer, J. P. e Allen, N. J. (1997). Commitment in the workplace: Theory, research, and application. Thousand Oaks: CA, Sage.

Meyer, J. P., Allen, N. J. e Smith, C. A. (1993). Commitment to organizations and occupations: Extension and test of a threecomponent conceptualization. Journal of Applied Psychology, 78(4), 538-551.

Meyer, J. P. et al (1993). Development of organizational commitment during the first year of employment: A longitudinal study of pre- and post-entry influences. Journal of Management, 17, 717-733.

Meyer, J. P. e Herscovitch, L. (2001). Commitment in the workplace: Toward a general model. Human Resource Management Review, 11, 299-326.

Moreira, M. et al (2003). Estilos de gestão de conflitos nas organizações: uma contribuição para a prática construtiva da resolução de conflitos: In recursos humanos magazine. Lisboa: Editora RH, Lda, p. $48-45$.

Morrow, P. C. e Wirth, R. E. (1989). Work commitment among salaried professionals. Journal of Vocational Behavior, 34, 40-56.

Nursing. (2000). Modos de resolução construtiva. Lisboa. ISSN 087 6196. p. 28-30.

Penley, L. e Gould, S., 1988. Etzioniees model of organizational involvement: a perspective for understanding commitment to organizations. Journal of Organizational Behavior, 9, 43-59.

Pruit, D. e Rubin, J. (1986). Social conflict: escalation, stalemate and settlement. New Work: Random House.

Rahim, M. A. (1986). Referent Role and Styles of Handling Interpersonal Conflict. The Journal of Social Psychology, 126 (1), p.79-86.

Rahim, M. (2001). Managing conflict in organizations. Westport: Quorum Books.

Rahim, M. (2002). Toward a Theory of Managing Organizational Conflict. International Journal of Conflict Management, 3 (13), 206235.

Rahim, M. (2003). Rahim Organizational Conflict Inventories Experimental Edition. Palo Alto: Consulting Psychologist Press.

Rego, A. (2003). Empenhamento organizacional e ausência psicológica: Afinal quantas dimensões? Revista de Administração de Empresas, 43 (4) , 25-35. 
Rego, A. e Souto, S. (2002). Comprometimento organizacional: Um estudo luso-brasileiro sobre a importância da justiça. Anais do XXVI Encontro da ANPAD (Associação Nacional dos Programas de Pós-Graduação em Administração), Salvador, Brasil, 22-25 Setembro.

Robbins SP. (2002). Comportamento organizacional. $9^{a}$ ed. São Paulo: Prentice Hall.

Santos D. M. N. (2008). A influência do empenhamento organizacional e profissional dos enfermeiros nas estratégias de resolução de conflito. Lisboa: ISCTE, 2008. Tese de mestrado. Disponível em www: http://hdl.handle.net/10071/1001.

Thomas, K. (1992). Conflict and negotiation processes in Organizations.In Handbook of Industrial and Organizational Psychology. Palo Alto: Consulting Psychologist Press.

Tjosvold, D. et al (2000).Conflict management, efficacy and performance in organizational teams. Personnel Psychology, 53 (3), 625-642. 